\title{
Algebraically hyperbolic manifolds have finite automorphism groups
}

\author{
Fedor Bogomolov, Ljudmila Kamenova 1 , Misha Verbitsky 2
}

\begin{abstract}
A projective manifold $M$ is algebraically hyperbolic if there exists a positive constant $A$ such that the degree of any curve of genus $g$ on $M$ is bounded from above by $A(g-1)$. A classical result is that Kobayashi hyperbolicity implies algebraic hyperbolicity. It is known that Kobayashi hyperbolic manifolds have finite automorphism groups. Here we prove that, more generally, algebraically hyperbolic projective manifolds have finite automorphism groups.
\end{abstract}

\section{Introduction}

J.-P. Demailly introduced an algebraic analogue for the analytic notions of hyperbolicity in De. Using the classical Gauss-Bonnet formula he showed that every compact complex Kobayashi hyperbolic manifold is also algebraically hyperbolic. Demailly conjectured that for complex projective manifolds the two notions, Kobayashi hyperbolicity and algebraic hyperbolicity, coincide.

Kobayashi proved that Kobayashi hyperbolic manifolds have only finite order automorphisms, [Ko. In [KV] L. Kamenova and M. Verbitsky proved that algebraically hyperbolic projective hyperkähler manifolds have finite groups of automorphisms. The general expectation is that all projective hyperkähler manifolds are algebraically non-hyperbolic.

Theorem 1.1: (Kamenova, Verbitsky) Let $M$ be an algebraically hyperbolic projective hyperkähler manifold. Then $M$ has finite group of automorphisms.

Here we generalize the result above and show that all algebraically hyperbolic projective manifolds have finite groups of automorphisms.

Theorem 1.2: The group Aut $(M)$ of automorphisms of an algebraically hyperbolic manifold $M$ is finite.

The idea behind the proof of our main theorem is the following. We consider the induced action of the automorphism group $\operatorname{Aut}(M)$ on the cohomology $H^{1,1}(M, \mathbb{R})$. From the Hodge-Riemann relations it follows that the image of $\operatorname{Aut}(M)$ in $G L\left(H^{1,1}(M, \mathbb{R})\right)$ has to preserve some rational Kähler class. Furthermore, we consider the case when the image of $\operatorname{Aut}(M)$ in $\operatorname{Aut}\left(\operatorname{Pic}^{0}(M)\right)$ is infinite. Using the Albanese map we produce a subvariety of $M$ that admits

\footnotetext{
${ }^{1}$ Partially supported by a grant from the Simons Foundation/SFARI (522730, LK)

2 Partially supported by the Russian Academic Excellence Project '5-100', CNPq Process 313608/2017-2 and FAPERJ E-26/202.912/2018.
} 
self-isogenies of arbitrarily high order, thus giving curves of constant genus and arbitrary high degree in this subvariety, i.e., we obtain an algebraically nonhyperbolic subvariety. This contradiction implies that the image of $\operatorname{Aut}(M)$ in $\operatorname{Aut}\left(\operatorname{Pic}^{0}(M)\right)$ is finite. If we assume that $\operatorname{Aut}(M)$ is infinite, there would be an infinite subgroup $\Gamma$ acting trivially on $\operatorname{Aut}(\operatorname{Pic}(M))$. It fixes an ample line bundle $L \in \operatorname{Pic}(M)$. The action of $\Gamma$ on $\mathbb{P} H^{0}(M, L)^{*}$ preserves the image of the projective embedding $M \hookrightarrow \mathbb{P} H^{0}(M, L)^{*}$. Let $\bar{\Gamma}$ be the Zariski closure of $\Gamma$ in $P G L\left(H^{0}(M, L)^{*}\right)$. Then the orbits of $\bar{\Gamma}$ are positive dimensional, which is a contradiction, because $\operatorname{Aut}(M)$ is discrete when $M$ is algebraically hyperbolic.

\section{Brody curves, Kobayashi and algebraic hyper- bolicity}

In this section let $M$ be a compact complex manifold. We introduce some basic hyperbolicity notions that can be found in $[\mathrm{Ko}]$ and $[\mathrm{De}$. Brody introduced the Brody curves in $[\mathrm{Br}$.

\subsection{Kobayashi pseudometric and Brody curves}

Definition 2.1: A pseudometric on $M$ is a function $d: M \times M \longrightarrow \mathbb{R} \geqslant 0$ which is symmetric: $d(x, y)=d(y, x)$ and satisfies the triangle inequality $d(x, y)+$ $d(y, z) \geqslant d(x, z)$.

Remark 2.2: Let $\mathfrak{D}$ be a set of pseudometrics on $M$. Then $d_{\max }(x, y):=$ $\sup _{d \in \mathfrak{D}} d(x, y)$ is also a pseudometric.

Definition 2.3: The Kobayashi pseudometric $d_{M}$ on a complex manifold $M$ is $d_{M}:=d_{\max }$ for the set $\mathfrak{D}$ of all pseudometrics on $M$ such that any holomorphic map from the Poincaré disk to $M$ is distance-decreasing.

Remark 2.4: The definition above is equivalent to the standard definition that the Kobayashi distance between points $x, y \in M$ is the infimum of the Poincaré distance over all sets of Poincaré disks connecting $x$ to $y$.

Definition 2.5: A manifold $M$ is called Kobayashi hyperbolic if the Kobayashi pseudometric $d_{M}$ is non-degenerate.

Definition 2.6: Let $M$ be a complex Hermitian manifold. A Brody curve is a non-constant holomorphic map $f: \mathbb{C} \longrightarrow M$ such that $|d f| \leqslant C$ for some constant $C$. Here $|d f|$ is understood as an operator norm of $d f: T_{z} \mathbb{C} \longrightarrow T M$, where $\mathbb{C}$ is equipped with the standard Euclidean metric.

Definition 2.7: Let $\left(\Delta_{r}, g_{r}\right)$ be a disk of radius $r$ in $\mathbb{C}$ with the Poincaré metric $g_{r}$, rescaled in such a way that the unit tangent vector to 0 has length 1 . A 
Brody map to a Hermitian complex manifold $M$ is a map $f: \Delta_{r} \longrightarrow M$ such that $|d f| \leqslant 1$ (here the operator norm is taken with respect to the Poincaré metric on $\left.\Delta_{r}\right)$ and $|d f|(z)=1$ at $z=0$.

Lemma 2.8: Let $f_{r}: \Delta_{r} \longrightarrow M$ be a sequence of Brody maps with $r \longrightarrow \infty$. Then $\left\{f_{r}\right\}$ has a subsequence which converges uniformly to a Brody curve $f$ satisfying $|d f|(z)=1$ at $z=0$.

Proof. Let $r_{1}<r_{2}$. The identity map $\tau:\left(\Delta_{r_{1}}, g_{1}\right) \longrightarrow\left(\Delta_{r_{2}}, g_{2}\right)$ is 1Lipschitz. Indeed, it is 1-Lipschitz with respect to the usual Poincaré metric: $\tau^{*}\left(r_{2}^{-2} g_{2}\right) \leqslant r_{1}^{-2} g_{1}$. Since $r_{1}<r_{2}$, this gives $\tau^{*} g_{2} \leqslant g_{1}$. Restricted on any disk $\Delta_{R}$, the family $\left\{f_{r}, r>R\right\}$ is a normal family (since it is Lipschitz), hence it has a subsequence which converges uniformly to a Lipschitz map. Since a uniform limit of holomorphic maps is holomorphic, the family $\left\{\left.f_{r}\right|_{\Delta_{R}}, r>R\right\}$ converges to a holomorhic map on $\varphi_{R}: \Delta_{R} \longrightarrow M$. The map $\varphi_{R}$ is Lipschitz with respect to all metrics $g_{r}, r>R$. Since $\lim _{r \rightarrow \infty} g_{r}$ is the standard Euclidean metric $g_{\infty}$, the map $\varphi_{R}$ is Lipschitz with respect to $g_{\infty}$. The sequence $\lim _{R \longrightarrow \infty} \varphi_{R}$ converges to a holomorphic Lipschitz map $\mathbb{C} \longrightarrow M$. Since all $f_{r}$ and $\varphi_{R}$ satisfy $\left|d \varphi_{R}\right|(z)=1$ at $z=0$, the same is true for the limit.

A classical result of Brody states that a compact complex manifold $M$ is Kobayashi hyperbolic if and only if there are no non-constant holomorphic maps from $\mathbb{C}$ to $M$.

Theorem 2.9: (Brody's lemma) Let $M$ be a compact complex manifold which is not Kobayashi hyperbolic. Then $M$ contains a Brody curve.

Proof: Let us equip $M$ with a Hermitian metric $h$. If $|d f|(0) \leqslant C$ for any holomorphic map $\left(\Delta_{1}, g_{1}\right) \longrightarrow M$, then the Kobayashi pseudometric satisfies $d_{K} \geqslant C^{-1} h$, and $M$ is Kobayashi hyperbolic. If this quantity is non-bounded, we can always rescale the disc to obtain a map $f_{r}:\left(\Delta_{r}, g_{r}\right) \longrightarrow M$ with $r=|d f|(0)$, and then $\left|d f_{r}\right|(0)=1$. Then Brody's lemma follows from Lemma 2.8 and the following lemma.

Lemma 2.10: Let $M$ be a compact Hermitian manifold, and $\psi_{r}:\left(\Delta_{r}, g_{r}\right) \longrightarrow M$ a sequence of holomorphic maps satisfying $\left|d \psi_{r}\right|(0) \geqslant 1, r \longrightarrow \infty$. Then there exists a sequence of Brody maps $f_{s}:\left(\Delta_{s}, g_{s}\right) \longrightarrow M$, with $s \longrightarrow \infty$.

Proof. We need to construct a sequence of Brody maps, which are 1Lipschitz maps $f_{s}:\left(\Delta_{s}, g_{s}\right) \longrightarrow M$, with $\left|d f_{s}\right|(0)=1$. The identity map

$$
\Psi_{r-\varepsilon, r}:\left(\Delta_{r-\varepsilon}, g_{r-\varepsilon}\right) \longrightarrow\left(\Delta_{r}, g_{r}\right)
$$

is 1-Lipschitz, and satisfies

$$
\lim _{z \longrightarrow \partial \Delta_{r-\varepsilon}}\left|d \Psi_{r-\varepsilon, r}\right|(z)=0 .
$$


Let $u:=r-\varepsilon$ and $\widetilde{f}_{u}:=\Psi_{r-\varepsilon, r} \circ \psi_{r}$ be a restriction of $f_{r}$ to the disk $\left(\Delta_{r-\varepsilon}, g_{r-\varepsilon}\right)$. Then $f_{u}$ is also Lipschitz and $\left|d \widetilde{f}_{u}\right|$ reaches maximum at a point $z_{u}$ somewhere inside the disk $\Delta_{u}$. Applying an appropriate holomorphic isometry of $\Delta_{u}$, we may assume that $\left|d \widetilde{f}_{u}\right|(z)$ takes maximum $C_{u} \geqslant 1$ for $z=0$. Rescaling $\widetilde{f}_{u}$, and setting $s:=C_{u} u$, we obtain a map $f_{s}: \Delta_{s} \longrightarrow M$ which is 1-Lipschitz and satisfies $\left|d f_{s}\right| \leqslant 1,\left|d f_{s}\right|(0)=1$.

Definition 2.11: Let $M$ be a projective manifold. We say that $M$ is algebraically hyperbolic if there exists a constant $A>0$ such that for any curve $C \subset M$ of geometric genus $g$ one has $\operatorname{deg} C<A(g-1)$.

Remark 2.12: Algebraically hyperbolic manifolds contain no elliptic nor rational curves.

Using Gauss-Bonnet in De Demailly proved that Kobayashi hyperbolicity implies algebraic hyperbolicity. Here we'll give a slightly different proof of this well known result. The converse implication, "algebraically hyperbolicity implies Kobayashi hyperbolicity", was conjectured by J.-P. Demailly who introduced the notion of algebraic hyperbolicity in De.

Theorem 2.13: (Demailly, De] Kobayashi hyperbolicity implies algebraic hyperbolicity.

Proof. Let $C \subset M$ be a curve in a Kobayashi hyperbolic manifold. The genus 0 and 1 cases are ruled out by Brody's Lemma, Theorem 2.9. Then genus $g=g(C)$ is greater than 1 , and its universal cover is the disk $\Delta$. Denote by $\varphi_{C}: \Delta \longrightarrow M$ the universal covering map. The volume of $C$ with respect to the Fubini-Study metric on $M$ is $\operatorname{deg} C$, and its volume with respect to the Poincaré metric is $\int_{C} c_{1}\left(T^{*} C\right)=2 \pi(2 g-2)$. Therefore, $\left|\varphi_{C}\right|>\frac{\operatorname{deg} C}{2 \pi(2 g-2)}$ somewhere on $\Delta$. Notice that $M$ is not algebraically hyperbolic if and only if there is a sequence $\left\{C_{i}\right\}$ of curves in $M$ with $\lim _{i \longrightarrow \infty} \frac{\operatorname{deg} C_{i}}{2 \pi\left(2 g\left(C_{i}\right)-2\right)} \longrightarrow \infty$. Suppose that $M$ is not algebraically hyperbolic. This gives a sequence $\varphi_{C_{i}}: \Delta_{1} \longrightarrow M$ with $\left|\varphi_{C_{i}}\right|>\frac{\operatorname{deg} C_{i}}{2 \pi\left(2 g\left(C_{i}\right)-2\right)}$ somewhere on $\Delta$. Replacing $\Delta_{1}$ by $\Delta_{1-\varepsilon}$ as above if necessary, we may assume that $\left|\varphi_{C_{i}}\right|$ reaches its maximum somewhere on $\Delta_{1}$. Applying an isometry of $\Delta_{1}$, we may assume that $\left|\varphi_{C_{i}}\right|$ reaches its maximum $R_{i}$ in $0 \in \Delta_{1}$. Rescaling $\varphi_{C_{i}}$ by $R_{i}$, we obtain a sequence of disks $\widetilde{\varphi}_{C_{i}}(z)=$ $\varphi_{C_{i}}\left(z / R_{i}\right): \Delta_{R_{i}} \longrightarrow M$, giving a Brody curve by Lemma 2.8

\subsection{Algebraic Kobayashi metric}

Definition 2.14: The Kobayashi pseudometric $d_{K}$ is defined as the infimum of the path metric on connected chains of holomorphic disks equipped with the Poincaré metrics. Define the algebraic Kobayashi pseudometric $d_{A}$ using the Poincaré metric on connected chains of algebraic curves instead of the disks. 
Remark 2.15: This metric clearly satisfies $d_{A} \geqslant d_{K}$. In [DLS] Demailly, Lempert and Shiffman prove that $d_{A}=d_{K}$ for any quasi-projective manifold, i.e., one can compute the Kobayashi pseudodistance by means of chains of algebraic curves. Also, algebraic hyperbolicity follows if $d_{A}$ is non-degenerate; this is proven by reverse-engineering the argument we used to prove that algebraic hyperbolicity is implied by Kobayashi hyperbolicity. It is not clear, however, if algebraic hyperbolicity implies the non-degeneracy of $d_{A}=d_{K}$.

Question 2.16: Is $d_{A}$ always non-degenerate for algebraically hyperbolic manifolds?

Remark 2.17: Note that the group $\operatorname{Aut}(M)$ of holomorphic automorphisms of a complex manifold $M$ with $d_{A}$ non-degenerate is necessarily compact. Indeed, any holomorphic automorphism of $M$ is an isometry of $\left(M, d_{A}\right)$, and the group of isometries of a compact metric space is compact. On the other hand, a uniform limit of holomorphic maps is holomorphic, hence compactness of the isometry group implies compactness of $\operatorname{Aut}(M)$. Since an algebraically hyperbolic manifold has discrete group of holomorphic automorphisms by Proposition 2.19, the group $\operatorname{Aut}(M)$ is finite. Then an affirmative answer to Question 2.16 would imply the main statement of this paper: finiteness of $\operatorname{Aut}(M)$ for algebraically hyperbolic $M$.

Remark 2.18: For any $g$ there are complex elliptic fibrations over $\mathbb{P}^{1}$ without multiple fibers where all horizontal curves (i.e., curves surjecting onto $\mathbb{P}^{1}$ under the projection) have genus greater than $g$ (Remark 6.11 in [BT]). The Kobayashi pseudometric on such surfaces is trivial but there are no elliptic or rational curves connecting points in different fibers. This example illustrates the difference in obtaining the Kobayashi pseudometric on the surface from mapping complex surfaces and algebraic curves. We can connect any two points in the surface by the image of a complex line, as shown in $\mathrm{BL}$. However, we need to take a limit over all chains of algebraic curves connecting points in different fibers in order to obtain the Kobayashi pseudometric using only algebraic curves.

\subsection{Automorphisms of hyperbolic manifolds}

In the rest of the section we summarize some facts about the automorphism group of hyperbolic manifolds.

Proposition 2.19: The group of automorphisms of an algebraically hyperbolic manifold $M$ is discrete.

Proof. The group of automorphisms $G$ of a projective manifold $M$ is a complex Lie group. If its connected component $G^{0}$ is non-trivial, this gives a holomorphic map $\varphi: G^{0} \longrightarrow M$, depending on the choice of a point. Any connected complex algebraic Lie group is an extension of an affine group and an 
abelian variety. Affine algebraic groups are rational varieties, hence the orbit of an affine algebraic group is unirational and covered by rational curves. An abelian variety is not algebraically hyperbolic, because an abelian variety of dimension $n$ admits a self-isogeny of order $m^{n}$ mapping a curve $C$ of genus $g$ to a curve of genus $g$ and degree $m^{n} \operatorname{deg} C$. The same argument shows that any positive-dimensional orbit of a compact complex commutative Lie group is not algebraically hyperbolic. Notice that the image of an algebraically nonhyperbolic manifold is also not algebraically hyperbolic. Therefore, $M$ cannot be algebraically hyperbolic.

An even stronger statement is true for Kobayashi hyperbolic manifolds.

Proposition 2.20: (Kobayashi, $\mathrm{Ko}$ ) The group of automorphisms of a compact Kobayashi hyperbolic manifold $M$ is finite.

Proof. The group $G$ of automorphisms of $M$ is closed in its group of isometries under the Kobayashi pseudometric. The group of isometries of a compact metric space is compact, hence $G$ has only finitely many connected components. Finally, $\operatorname{dim} G^{0}=0$ as shown in Proposition 2.19, i.e., $G$ is discrete and compact, therefore finite.

\section{Main Results}

In this section $M$ is a compact complex projective manifold. For the classical notions of the Picard scheme and the Albanese variety we refer the readers to the survey manuscript $\underline{\mathrm{K}}]$.

Proposition 3.1: Let $M$ be a complex projective manifold, $\operatorname{dim}_{\mathbb{C}} M=n$. Suppose that the image of $\operatorname{Aut}(M)$ in $G L\left(H^{1,1}(M, \mathbb{R})\right)$ does not preserve any rational Kähler class. Then $M$ is not algebraically hyperbolic.

Proof. Assume $M$ is algebraically hyperbolic. Let $\omega$ be a rational Kähler class which has an infinite Aut $(M)$-orbit. Replacing $\omega$ by $N \omega$, from the $(1,1)$ theorem we may assume that $\omega$ is a class of a hyperplane section. Then $\omega^{n-1}=$ $[C]$ is the fundamental class of a smooth complex curve $C \subset M$ from Bertini's theorem. Let $f_{i}(\omega)$ be in the orbit of $\omega$, which is infinite by assumption. Then

$$
\operatorname{deg}_{\omega} f_{i}(C)=\int_{M} \omega \wedge\left(f_{i}(\omega)\right)^{n-1}=\int_{M} f_{i}^{-1}(\omega) \wedge \omega^{n-1} .
$$

Since the genus of $f_{i}(C)$ is constant, from algebraic hyperbolicity we obtain $\int_{M} f_{i}(\omega) \wedge \omega^{n-1}<A$ for some constant $A>0$.

Let $|\cdot|$ denote the positive-definite Hodge-Riemann metric on $H^{p, q}(M, \mathbb{R})$, that is, the metric defined as $\eta, \eta^{\prime} \longrightarrow-(-1)^{l}(\sqrt{-1})^{p-q} \int_{M} \eta \wedge \overline{\eta^{\prime}} \wedge \omega^{n-p-q}$, where $\eta, \eta^{\prime}$ are $(p, q)$-forms which belong to a weight $l$ representation of $\mathfrak{s l}(2)$ associated with the Lefschetz $\mathfrak{s l}(2)$-action. Replace the sequence $\frac{f_{i}(\omega)}{\left|f_{i}(\omega)\right|}$ of points in the 
sphere $S \subset H^{1,1}(M, \mathbb{R})$ by a converging subsequence, and let $R$ be its limit. Since the sequence $f_{i}(\omega)$ is infinite, distinct and integral, one has $\lim _{i \rightarrow \infty}\left|f_{i}(\omega)\right|=$ $\infty$. Then $\int_{M} f_{i}(\omega) \wedge \omega^{n-1}<A$ implies that $\int_{M} R \wedge \omega^{n-1}=0$. By the HodgeRiemann relations, this gives $\int_{M} R \wedge R \wedge \omega^{n-2}=-|R|^{2}=-1$, hence

$$
\lim _{i} \frac{\int_{M} f_{i}(\omega) \wedge f_{i}(\omega) \wedge \omega^{n-2}}{\left|f_{i}(\omega)\right|^{2}}=-1 .
$$

This is a contradiction, because $f_{i}(\omega)$ is Kähler, hence $\int_{M} f_{i}(\omega) \wedge f_{i}(\omega) \wedge \omega^{n-2}>$ 0 .

Proposition 3.2: Let $M$ be a complex projective manifold. Suppose that $\operatorname{Aut}(M)$ is infinite, but the image of $\operatorname{Aut}(M)$ in $\operatorname{Aut}(\operatorname{Pic}(M))$ is finite. Then $M$ is not algebraically hyperbolic.

Proof. From the assumption in the proposition we obtain that an infinite subgroup $\Gamma \subset \operatorname{Aut}(M)$ acts trivially on $\operatorname{Pic}(M)$. Then it fixes a very ample line bundle $L \in \operatorname{Pic}(M)$. Therefore, $\Gamma$ acts on $\mathbb{P} H^{0}(M, L)^{*}$ preserving the image of the projective embedding $M \longrightarrow \mathbb{P} H^{0}(M, L)^{*}$. Let $G=\bar{\Gamma}$ be the Zariski closure of $\Gamma$ in $P G L\left(H^{0}(M, L)^{*}\right)$. Since $M$ is Zariski closed and $\Gamma$-invariant, it is $G$ invariant. Since $\Gamma$ acts on $M$ with infinite orbits, the orbits of the $G$-action on $M$ are positive-dimensional. This is impossible, because $\operatorname{Aut}(M)$ is discrete as shown in Proposition 2.19.

Remark 3.3: Notice that if the image $\mathfrak{G}$ of $\operatorname{Aut}(M)$ in $G L\left(H^{1,1}(M, \mathbb{R})\right)$ does not preserve any rational Kähler classes, then $\mathfrak{G}$ is infinite. Otherwise, take a finite orbit of a Kähler class and notice that its geometric center is an $\operatorname{Aut}(M)$ invariant Kähler class, because the convex hull of a set of Kähler classes lies inside the Kähler cone.

Remark 3.4: When $M$ is projective and the image $\mathfrak{G}$ of $\operatorname{Aut}(M)$ in $G L\left(H^{1,1}(M, \mathbb{R})\right)$ is finite, we could take a $\mathfrak{G}$-orbit of a rational Kähler class, and its geometric center is also rational. Therefore, any projective manifold $M$ with $\mathfrak{G}$ finite admits an $\operatorname{Aut}(M)$-invariant integer Kähler class.

Proposition 3.5: Let $M$ be a complex projective manifold. Suppose that the image of $\operatorname{Aut}(M)$ in $G L\left(H^{1,1}(M, \mathbb{R})\right)$ is finite, but its image in $\operatorname{Aut}\left(\operatorname{Pic}^{0}(M)\right)$ is infinite. Then $M$ is not algebraically hyperbolic.

Proof. Consider the orbit $\operatorname{Aut}(M) \cdot \alpha$ of a Kähler class $\alpha$. Its geometric center gives an $\operatorname{Aut}(M)$-invariant Kähler class $\omega$, because the convex envelope of a set of Kähler classes lies in the Kähler cone, as in Remark 3.3.

The Albanese manifold $\operatorname{Alb}(M)=H^{0}\left(M, \Omega_{M}^{1}\right)^{*} / H_{1}(M, \mathbb{Z})$ admits a natural Aut $(M)$-invariant flat Kähler metric induced by the Hodge-Riemann form on $H^{1}(M, \mathbb{C})$. Since $\operatorname{Aut}(M)$ acts on $\operatorname{Alb}(M)$ by isometries, the image $\mathfrak{T}$ of $\operatorname{Aut}(M)$ in $\operatorname{Aut}(\operatorname{Alb}(M))$ is compact. The connected component of $\operatorname{Aut}(\operatorname{Alb}(M))$ is a 
subgroup $\operatorname{Par}(\operatorname{Alb}(M))=\operatorname{Alb}(M) \subset \operatorname{Aut}(\operatorname{Alb}(M))$ acting on the torus $\operatorname{Alb}(M)$ by parallel transport. Since $\mathfrak{T}$ is compact, its image in the discrete group $\frac{\operatorname{Aut}(\operatorname{Alb}(M))}{\operatorname{Par}(\operatorname{Alb}(M))}$ is finite. Let $\Gamma \subset \mathfrak{T}$ be the kernel of the map $\mathfrak{T} \longrightarrow \frac{\operatorname{Aut}(\operatorname{Alb}(M))}{\operatorname{Par}(\operatorname{Alb}(M))}$. This is a finite index subgroup in $\mathfrak{T}$. Therefore, $\Gamma$ is infinite.

Since $\Gamma$ is infinite, its closure $T \subset \operatorname{Par}(\operatorname{Alb} M)$ is positive-dimensional. Take a smooth fiber $\mathrm{Alb}^{-1}(x)$ over $x \in \operatorname{Alb}(M)$. The general fiber of the real analytic map $\operatorname{Alb}^{-1}(T \cdot x) \stackrel{\pi}{\longrightarrow} T \cdot x$ is smooth. Since all fibers of $\pi$ exist in dense families, all fibers of $\pi$ are smooth. Therefore, $\pi$ is a locally trivial fibration with isomorphic fibers. Consider the Zariski closure $T_{1}$ of $T$, which is a compact commutative Lie group, that is, the compact complex torus. This gives an isotrivial fibration $\mathrm{Alb}^{-1}(X) \stackrel{\pi_{1}}{\longrightarrow} X$, where $X$ is an orbit of $T_{1}$.

Isotrivial fibrations over $T_{1}$ with fiber $F$ are classified by $H^{1}\left(T_{1}, \operatorname{Aut}(F)\right)$. The variety $F \subset M$ may be assumed to be an algebraically hyperbolic manifold with $\operatorname{dim} F<\operatorname{dim} M$. Using induction on dimension, we may assume that $\operatorname{Aut}(F)$ is finite.

The first cohomology of a torus with coefficients in a finite group $A$ is the same as an $A$-valued local system. Therefore, it becomes trivial after an appropriate finite covering. Then $\pi_{1}$ becomes a trivial fibration after passing to a finite covering $Y \longrightarrow X$, giving a decomposition $\widetilde{\mathrm{Alb}^{-1}(X)}=F \times Y$. This manifold admits self-isogenies of arbitrary high order, giving curves of constant genus and arbitrary high degree in $\widetilde{\mathrm{Alb}^{-1}(X)}$ and its finite quotient $\mathrm{Alb}^{-1}(X)$. Therefore, $\mathrm{Alb}^{-1}(X) \subset M$ is not algebraically hyperbolic, and $M$ is algebraically nonhyperbolic.

Using the above results we can prove the main result of this paper.

Theorem 3.6: The group $\operatorname{Aut}(M)$ of automorphisms of an algebraically hyperbolic manifold $M$ is finite.

Proof. Assume $\operatorname{Aut}(M)$ is infinite. If the image $\mathfrak{G}$ of the group $\operatorname{Aut}(M)$ in $G L\left(H^{1,1}(M, \mathbb{R})\right)$ does not preserve any rational Kähler class, $\mathfrak{G}$ is infinite by Remark 3.4. Then we would get a contradiction by Proposition 3.1. If the image of $\operatorname{Aut}(M)$ in $\operatorname{Aut}(\operatorname{Pic}(M))$ is finite, we get a contradiction by Proposition 3.2. and if its image in $\operatorname{Aut}(\operatorname{Pic}(M))$ is infinite, we get a contradiction by Proposition 3.5.

To sum the argument up: we consider the image $\mathfrak{G}$ of the group $\operatorname{Aut}(M)$ in $G L\left(H^{1,1}(M, \mathbb{R})\right)$. If it is finite, we have an integer Kähler class which is $\mathfrak{G}$-invariant. If $\operatorname{Aut}(M)$ acts with finite image on the Albanese variety of $M$, this class gives an $\operatorname{Aut}(M)$-invariant polarization, hence $\operatorname{Aut}(M)$ is a linear algebraic group; its connected part is trivial because the orbits of a connected linear algebraic group are rational.

If $\mathfrak{G}$ is finite, but $\operatorname{Aut}(M)$ acts with infinite image on the Albanese variety of $M$, the closure of an orbit of $\operatorname{Aut}(M)$ contains a torus, also contradicting hyperbolicity. 
Finally, if $\mathfrak{G}$ is infinite, be obtain curves of bounded genus and arbitrary degree using the action of $\mathfrak{G}$ on cohomology.

Acknowledgments. Most of the results in this paper were finalized during the Komplexe Analysis Oberwolfach Workshop in 2017. The second and thirdnamed authors are grateful to the Oberwolfach organizers and staff for their hospitality. The first-named author has been funded by the Russian Academic Excellence Project '5-100' and acknowledges support by Simons travel grant and by the EPSRC program grant EP/M024830. We are thankful to the referee for many valuable comments and corrections to the earlier version.

\section{References}

[BT] Bogomolov, F., Tschinkel, Y., Algebraic varieties over small fields, Special semester on Arithmetic geometry at Centro Ennio De Giorgi, CRM Series 4 (2007) 73-91.

[Br] Brody, R., Compact manifolds and hyperbolicity, Trans. Amer. Math. Soc. 235 (1978) 213-219.

[BL] Buzzard, G.; Lu, S. S.-Y., Algebraic surfaces holomorphically dominable by $\mathbb{C}^{2}$, Invent. Math. 139 (2000), no. 3, 617-659.

[De] Demailly, J.-P., Algebraic criteria for Kobayashi hyperbolic projective varieties and jet differentials, Proc. Symp. Pure Math., Vol 62.2 (1997) $285-360$.

[DLS] Demailly, J.-P., Lempert, L., Shiffman, B., Algebraic approximations of holomorphic maps from Stein domains to projective manifolds, Duke Math. J. 76 (1994) 333-363.

[GH] Griffiths, Phillip; Harris, Joe; Principles of Algebraic Geometry, WileyInterscience, New York, 1978.

[KV] Kamenova, L., Verbitsky, V., Algebraic non-hyperbolicity of hyperkähler manifolds with Picard rank greater than one, NYJM 23 (2017) 489-495.

[Kl] Kleiman, S., The Picard scheme, Fundamental algebraic geometry, Math. Surveys Monog., 123, Providence, R.I., AMS (2005) 235-321.

[Ko] Kobayashi, S., Intrinsic distances, measures and geometric function theory, Bull. Amer. Math. Soc. 82, no. 3 (1976) 357 - 416.

Fedor A. Bogomolov

DePARTMENT OF Mathematics

Courant Institute, NYU

251 MERCER StreET

NEW YoRK, NY 10012, USA, 
bogomolov@cims.nyu.edu, also:

National Research University, Higher School of Economics, Moscow, Russia,

LJUDMILA KAMENOVA

Department of Mathematics, 3-115

STONY Brook UNIVERSITY

STONY Brook, NY 11794-3651, USA,

kamenova@math. sunysb.edu

Misha VERBITSKY

Instituto Nacional de Matemática Pura e Aplicada (IMPA)

Estrada Dona Castorina, 110

JARDIM BOTÂNICO, CEP 22460-320

Rio DE JANEIRO, RJ - BRASIL

ALSO:

LABORATORY of Algebraic GeOMETry,

National Research University Higher School of Economics,

Department of Mathematics, 6 Usacheva street, Moscow, Russia. 